\title{
CONSTRAINED REGULATOR PROBLEM FOR LINEAR UNCERTAIN SYSTEMS: CONTROL OF A PH PROCESS
}

\author{
FOUAD MESQUINE, FERNANDO TADEO, ABDELLATIF BENLAMKADEM
}

Received 8 November 2005; Accepted 14 February 2006

The regulator problem for linear uncertain continuous-time systems having control constraints is considered. Necessary and sufficient conditions of positive invariance of polyhedral domains are extended to the case of continuous-time uncertain systems. Robust constrained regulators are then derived. An application to the control of $\mathrm{pH}$ in a stirred tank is then presented. First, the uncertainty in the $\mathrm{pH}$ process is evaluated from firstprinciple models, then the design of a robust constrained regulator is presented. Simulation results show that this control law is easy to implement and that robust asymptotic stability and control admissibility are guaranteed.

Copyright (c) 2006 Fouad Mesquine et al. This is an open access article distributed under the Creative Commons Attribution License, which permits unrestricted use, distribution, and reproduction in any medium, provided the original work is properly cited.

\section{Introduction}

It is well known these last years that for physical, technological and/or security reasons, any real system is subject to operational limitations, which are introduced as constraints. The most encountered constraints are of saturation type, that is, limitations on the magnitude and/or derivative of certain variables, usually the control signal. Hence, the topic of designing control systems that maintain stability and performance in the presence of these constraints is a topic of continuing interest. There are several approaches proposed in the literature to solve this problem: the positive invariance concept $[2-5,8,9,21]$, the small and high gain concept [17], the $l_{1}$ norm optimization concept [11], predictive control techniques $[10,18]$, and other approaches $[16,27]$. From these approaches, the positive invariance approach is selected in this paper, because it gives simple methods to design constant state-feedback controllers, in both the continuous and the discrete-time cases, with symmetrical and nonsymmetrical constraints. The concept of positive invariance is involved in many control problems as constraints, robustness, pole assignment, optimization, and so forth. A literature overview about positively invariant sets in control theory is given in [9]. Recall that a subset, polyhedral or ellipsoidal, of the state space is

Hindawi Publishing Corporation

Mathematical Problems in Engineering

Volume 2006, Article ID 51874, Pages 1-15

DOI 10.1155/MPE/2006/51874 
said to be positively invariant with respect to the motion of a dynamic system if it has the useful property that if it contains the system state at some time, then the state will never leave that set in the future. This approach corresponds to constraint avoidance [16]: preventing the saturation, the closed-loop system stays in a region of linear behavior. Hence, theory of linear systems remains valid and then stability of the closed-loop constrained system is obtained by assigning the closed-loop spectrum in the stability region, that is, the left half-plane for continuous-time case and the unity disk for the discrete-time case.

On the other hand, due to the inherent nonlinear behavior of real systems, linearization techniques and approximations lead to uncertain linear models. These uncertainties are generally induced by the difference between the real behavior of the system and the behavior of the plant model used for control. Many progress has been accomplished, these last years, in the control methods to take into account these uncertainties. Several techniques have been proposed in the literature, grouped in the theory of robust control, for example, [32], the simultaneous presence of uncertainties and constraints in general physical systems, and have prompted many authors to combine the techniques of robust control and constrained control $[4,7]$. With the same goal and as an extension to the uncertain continuous-time case of [3], this paper is devoted in a first part to the robust constrained regulator problem for linear continuous-time systems. Robustness conditions for constrained state-feedback regulators are obtained. To do so, necessary and sufficient conditions of positive invariance of polyhedral domains with respect to motion of uncertain continuous-time systems are derived. The second part of this paper is devoted to the application of this robust control law to a neutralization process, namely a $\mathrm{pH}$-process.

During the last years, several approaches have been proposed in the literature to solve $\mathrm{pH}$ control problems, that is, process control problem where the main output variable is the $\mathrm{pH}$ of a solution. Both nonlinear and linear controller techniques have been proposed and proved on neutralization processes: nonlinear controllers should be the natural solution to $\mathrm{pH}$ control as the inherent nonlinear behavior of $\mathrm{pH}$ process.

For example, nonlinear controllers have been proposed by [22] using an internal model control strategy with a nonlinear adaptive model. Nonlinear controllers have also been studied in [31], and different researchers have also considered nonlinear predictive control techniques: the authors in [14] have proposed to use a neural predictive strategy to study in-line $\mathrm{pH}$ process, and the authors in [23] have studied the use of both static nonlinear element and linear dynamic elements of SISO Wiener model. Although nonlinear techniques have been proved successfully on real plants, the main difficulty remains the necessity of developing a model that represents adequately the $\mathrm{pH}$ process in any operating condition. To solve this problem, fuzzy controllers have also been proposed in the literature. For example, $[6,12,26]$ have presented different approaches to fuzzy $\mathrm{pH}$ control.

Although these nonlinear approaches have been successfully proved in simulation and on laboratory plants, the developed controllers are quite complex, so they are difficult to implement on real industrial process.

Also, most of these techniques do not give definite methods to cope with plant uncertainty: performance and stability might be degraded, even lost, if the working conditions are different than those used to design the controller. 
This paper proposes a solution to this problem based on designing linear controllers with guaranteed robustness characteristics even in the presence of limitations in the control signals. Simulation results will show the performance of this $\mathrm{pH}$ regulator in the presence of constraints and uncertainty. A first attempt of this approach was achieved in [19]. We must point out that the problem of uncertainty of $\mathrm{pH}$ processes has been also studied in the literature, in the context of robust control using multiple models in $[13,24,30]$.

The problem of robust $\mathrm{pH}$ control with control saturation has also been studied by the authors in [29] using a two-degrees-of-freedom structure and high-order linear controllers. As claimed above, the second part of this paper studies the use of a different method to solve this problem, to get a simpler control structure, easy to implement in existing control systems. This method is based on the approach presented in [20].

The paper is divided into two parts. A first part where the theoretical needed background is derived and is organized as follows: the studied problem is stated in Section 2. Section 3 is devoted to recall some useful definitions and results about positive invariance for continuous-time perfectly known systems. The extension to the uncertain case of these results is achieved in Section 4 enabling one to derive necessary and sufficient conditions of positive invariance of polyedra with respect to uncertain systems. Using these conditions is the key to derive robust controllers for linear uncertain systems with constrained inputs. The second part is devoted to the application of the previous results on a neutralization process, namely a $\mathrm{pH}$-process, and is organized as follows: Section 5 presents the model of the studied $\mathrm{pH}$ process together with simulation results. The effectiveness and the easy application of such control law for a complicated process is proved. Concluding remarks end this paper.

\section{Problem statement}

Consider the linear uncertain continuous-time system represented by the following statespace model:

$$
\dot{x}(t)=A\left(q_{A}(t)\right) x(t)+B\left(q_{B}(t)\right) u(t)
$$

where $x(t) \in \mathbb{R}^{n}$ denotes the state vector, $u(t) \in \Omega \subset \mathbb{R}^{m}$ is the control input. The control input is restricted (by saturation) to evolve in the following polyhedral set:

$$
\Omega=\left\{u(t) \in \mathbb{R}^{m} /-u_{\min } \leq u \leq u_{\max } ; u_{\min }, u_{\max } \in \operatorname{Int} \mathbb{R}_{+}^{m}\right\}
$$

where $q_{A}(t) \in \Gamma_{A} \subset \mathbb{R}^{p_{A}}$ (resp., $q_{B}(t) \in \Gamma_{B} \subset \mathbb{R}^{p_{B}}$ ) is the uncertain vector. $\Gamma_{A}$ and $\Gamma_{B}$ are compact convex sets including the origin in their interiors. These vectors $q_{A}(t)$ and $q_{B}(t)$ measure the uncertainty in the model, affecting the parameters of the matrices $A$ and $B$ 
4 Control of constrained uncertain systems: a $\mathrm{pH}$ process

as follows:

$$
\begin{aligned}
& A(q(t))=A_{o}+\sum_{h=1}^{p_{A}} A_{h} q_{A h}(t), \\
& B(q(t))=B_{o}+\sum_{h=1}^{p_{B}} B_{h} q_{B h}(t),
\end{aligned}
$$

where $q_{A h}(t)$ and $q_{B h}(t)$ represent the $h$ th component of vectors $q_{A}(t)$ and $q_{B}(t)$, respectively:

$$
\begin{aligned}
& q_{A}(t)=\left[\begin{array}{llll}
q_{A 1}(t) & q_{A 2}(t) & \cdots & q_{A p_{A}}(t)
\end{array}\right]^{T}, \\
& q_{B}(t)=\left[\begin{array}{llll}
q_{B 1}(t) & q_{B 2}(t) & \cdots & q_{B p_{B}}(t)
\end{array}\right]^{T} .
\end{aligned}
$$

Convexity and compactness of the set $\Gamma_{A}$ imply that there exist $\mu_{A}$ vertices $\nu_{i}, i=1, \ldots, \mu_{A}$ of $\Gamma_{A}$, such that every $q_{A} \in \Gamma_{A}$ can be written as a convex combination of $\nu_{i}$ as

$$
q_{A}=\sum_{i=1}^{\mu_{A}} \alpha_{i} \nu_{i} \quad \text { with } \sum_{i=1}^{\mu_{A}} \alpha_{i}=1,0 \leq \alpha_{i} \leq 1 \text { for } i=1, \ldots, \mu_{A} .
$$

The set $\Gamma_{B}$ is also convex and compact, so there also exist $\mu_{B}$ vertices $v_{j}, j=1, \ldots, \mu_{B}$ of $\Gamma_{B}$, such that every $q_{B} \in \Gamma_{B}$ can be written as a convex combination of $\nu_{j}$ as

$$
q_{B}=\sum_{j=1}^{\mu_{B}} \beta_{j} \nu_{j} \quad \text { with } \sum_{j=1}^{\mu_{B}} \beta_{j}=1,0 \leq \beta_{j} \leq 1 \text { for } j=1, \ldots, \mu_{B}
$$

and the matrix $A\left(q_{A}(t)\right)$ (resp., $\left.B\left(q_{B}(t)\right)\right)$ as

$$
A\left(q_{A}(t)\right)=\sum_{i=1}^{\mu_{A}} \alpha_{i}(t) A_{i} \quad\left(\operatorname{resp} ., B\left(q_{B}(t)\right)=\sum_{j=1}^{\mu_{B}} \beta_{j}(t) B_{j}\right)
$$

with $A_{i}=A\left(v_{i}\right)$ (resp., $\left.B_{j}=B\left(v_{j}\right)\right)$.

Assume that the pair $\left(A\left(q_{A}(t)\right), B\left(q_{B}(t)\right)\right)$ is controllable for every $q_{A} \in \Gamma_{A}$ and $q_{B} \in$ $\Gamma_{B}$.

The nominal system is given by

$$
\dot{x}(t)=A_{o} x(t)+B_{o} u(t) .
$$

The robust constrained regulator problem, which will be studied in this paper, is to find a state-feedback control law

$$
u(t)=F x(t), \quad F \in \mathfrak{R}^{m \times n} \text { with } \operatorname{rank}(F)=m
$$

which stabilizes the nominal system

$$
\dot{x}(t)=\left(A_{o}+B_{o} F\right) x(t)
$$


respecting the control constrained (2.2), such that the feedback system is robustly stable against parametric uncertainty, that is, the feedback stabilizes also the uncertain system (2.1).

Application of this control law, with respected control constraints, leads to the closedloop system

$$
\begin{aligned}
\dot{x}(t) & =\left(A\left(q_{A}(t)\right)+B\left(q_{B}(t)\right) F\right) x(t) \\
& =\sum_{j=1}^{\mu_{B}} \beta_{j} \sum_{i=1}^{\mu_{A}} \alpha_{i}\left(A_{i}+B_{j} F\right) \\
& =\sum_{j=1}^{\mu_{B}} \beta_{j} \sum_{i=1}^{\mu_{A}} \alpha_{i} A_{c}\left(\nu_{i j}\right) \\
& =A_{c}(q(t)),
\end{aligned}
$$

where $q(t) \in \Gamma=\left(\Gamma_{A} \times \Gamma_{B}\right)$ and $\nu_{i j}$ denotes the vertices of $\Gamma\left(i=1, \ldots, \mu_{A} ; j=1, \ldots, \mu_{B}\right)$.

Also, the proposed control law induces the following set of linear behavior in state space:

$$
\mathscr{D}=\left\{x(t) \in \mathbb{R}^{n} /-u_{\min } \leq F x \leq u_{\max }, u_{\min }, u_{\max } \in \operatorname{Int} \mathbb{R}_{+}^{m}\right\} .
$$

Note here that as long as the system states remain in the domain $\mathscr{D}$, the linear behavior is guaranteed. Otherwise, the closed-loop system is given by

$$
\dot{x}(t)=A\left(q_{A}(t)\right) x(t)+B\left(q_{B}(t)\right) \operatorname{sat}(F x(t)) .
$$

It is worth noticing that positive invariance of domain $\mathscr{D}$ given by (2.15) with respect to motion of the closed-loop system (2.11) is the cornerstone to derive robust constrained regulators.

\section{Preliminary results}

This section recalls some results that will be used later. Firstly, a definition of the positive invariance concept is given. To do so, let us consider the free uncertain system given by

$$
\dot{z}(t)=H(q(t)) z(t), \quad z(t) \in \mathbb{R}^{m}, q(t) \in \Gamma .
$$

Definition 3.1. A set $S$ of $R^{m}$ is positively invariant with respect to motion of the system (3.1) if for every $z_{o}\left(t_{o}\right) \in S, z\left(t, z_{o}, t_{o}\right) \in S$, for all $q(t) \in \Gamma$, for all $t>t_{o}$.

To give a solution to the stated problem, it is necessary to extend some results established without uncertainties to the case of uncertain systems. Let us recall these results, which consist of positive invariance conditions and a definition of a nonquadratic Lyapunov function. The extension will be studied afterwards. Positive invariance conditions are recalled as follows. 
6 Control of constrained uncertain systems: a $\mathrm{pH}$ process

Theorem 3.2 [3]. The domain D (2.15) is positively invariant with respect to system (2.10), if and only if there exist a matrix $H \in R^{m \times m}$, such that

$$
\begin{gathered}
F A+F B F=H F, \\
\tilde{H} U \leq 0,
\end{gathered}
$$

with

$$
\tilde{H}=\left[\begin{array}{ll}
H_{1} & H_{2} \\
H_{2} & H_{1}
\end{array}\right], \quad U=\left[\begin{array}{l}
u_{\max } \\
u_{\min }
\end{array}\right],
$$

where

$$
\begin{gathered}
H_{1}= \begin{cases}h_{l l} & \text { for } l=k, \\
h_{l k}^{+} & \text {for } l \neq k, h_{l k}^{+}=\operatorname{Sup}\left(h_{l k}, 0\right),\end{cases} \\
H_{2}= \begin{cases}0 & \text { for } l=k, \\
h_{l k}^{-} & \text {for } l \neq k, h_{l k}^{-}=\operatorname{Sup}\left(-h_{l k}, 0\right) .\end{cases}
\end{gathered}
$$

Secondly, to present a nonquadratic Lyapunov function, consider the autonomous linear continuous-time system

$$
\dot{z}(t)=H z(t)
$$

and the positive definite nonquadratic function

$$
V(z)=\operatorname{Max}_{i} \max \left\{\frac{z_{i}^{+}}{w_{i}^{1}}, \frac{z_{i}^{-}}{w_{i}^{2}}\right\},
$$

$V(z)$ must satisfy the following necessary and sufficient conditions in order to be a Lyapunov function for the system (3.5).

Theorem 3.3 [3]. The function $V(z)$ given by (3.6) is a Lyapunov function of system (3.5) if and only if

$$
\tilde{H} W \leq 0,
$$

where $W^{t}=\left[\begin{array}{ll}w_{1}^{t} & w_{2}^{t}\end{array}\right]$ with $w_{1}, w_{2} \in \operatorname{Int} \mathbb{R}_{+}^{m}$.

\section{Main result}

As previously stated, this section is devoted to the extension of the preliminary results presented in the previous section to the case of uncertain plants. This extension gives conditions for designing robust controllers in the presence of uncertainty and input limitations. The following lemma is fundamental to extend necessary and sufficient conditions for the positive invariance of the polyhedral domain:

$$
\mathscr{D}_{p}=\left\{z \in \mathbb{R}^{m} /-p_{2} \leq z: \leq p_{1}, p_{1}, p_{2} \in \operatorname{Int} \mathbb{R}_{+}^{m}\right\}
$$


with respect to motion of the autonomous uncertain system

$$
\dot{z}(t)=H(q(t)) z(t) .
$$

Lemma 4.1. The set $\mathscr{D}_{p}$ is positively invariant with respect to motion of system (4.2) if and only if it is positively invariant with respect to motion of system (4.2) at vertices $v_{i j}, i=$ $1, \ldots, \mu_{A}, j=1, \ldots, \mu_{B}$ of the set $\Gamma$.

Proof. Necessity. It is obvious that if the domain is positively invariant for every $q$, it is especially positively invariant at the vertices $v_{i j}, i=1, \ldots, \mu_{A}, j=1, \ldots, \mu_{B}$.

Sufficiency. Suppose that $\mathscr{D}_{P}$ is positively invariant with respect to motion of system (4.2) for $q=v_{i j}, i=1, \ldots, \mu_{A}, j=1, \ldots, \mu_{B}$, then

$$
\tilde{H}\left(v_{i j}\right) P \leq 0 \quad \text { with } P=\left[\begin{array}{ll}
p_{1}^{T} & p_{2}^{T}
\end{array}\right]^{T} .
$$

Decomposing this inequality, it is possible to write

$$
\begin{aligned}
& H_{1}\left(v_{i j}\right) p_{1}+H_{2}\left(v_{i j}\right) p_{2} \leq 0, \\
& H_{2}\left(v_{i j}\right) p_{1}+H_{1}\left(v_{i j}\right) p_{2} \leq 0,
\end{aligned}
$$

however according to the definition of matrices $H_{1}$ and $H_{2}$ given by (3.4), (4.4) can be written for $l=1, \ldots, m$ as

$$
h_{l l}\left(v_{i j}\right) p_{1}^{l}+\sum_{\substack{k=1 \\ k \neq l}}^{m} h_{l k}^{+}\left(v_{i j}\right) p_{1}^{k}+\sum_{\substack{k=1 \\ k \neq l}}^{m} h_{l k}^{-}\left(v_{i j}\right) p_{2}^{k} \leq 0,
$$

multiplying every inequality by $\alpha_{i}, \beta_{j}, i=1, \ldots, \mu_{A}, j=1, \ldots, \mu_{B}$, and summing on $i$ and $j$, leads to

$$
\sum_{j=1}^{\mu_{B}} \sum_{i=1}^{\mu_{A}} \beta_{j} \alpha_{i} h_{l l}\left(\nu_{i j}\right) p_{1}^{l}+\sum_{j=1}^{\mu_{B}} \sum_{i=1}^{\mu_{A}} \sum_{\substack{k=1 \\ k \neq l}}^{m} \beta_{j} \alpha_{i} h_{l k}^{+}\left(\nu_{i j}\right) p_{1}^{k}+\sum_{j=1}^{\mu_{B}} \sum_{i=1}^{\mu_{A}} \sum_{\substack{k=1 \\ k \neq l}}^{m} \beta_{j} \alpha_{i} h_{l k}^{-}\left(\nu_{i j}\right) p_{2}^{k} \leq 0
$$

which is equivalent to write

$$
\sum_{j=1}^{\mu_{B}} \sum_{i=1}^{\mu_{A}} \beta_{j} \alpha_{i} h_{l l}\left(v_{i j}\right) p_{1}^{l}+\sum_{\substack{k=1 \\ k \neq l}}^{m} \sum_{j=1}^{\mu_{B}} \sum_{i=1}^{\mu_{A}} \beta_{j} \alpha_{i} h_{l k}^{+}\left(v_{i j}\right) p_{1}^{k}+\sum_{\substack{k=1 \\ k \neq l}}^{m} \sum_{j=1}^{\mu_{B}} \sum_{i=1}^{\mu_{A}} \beta_{j} \alpha_{i} h_{l k}^{-}\left(v_{i j}\right) p_{2}^{k} \leq 0 .
$$

Using the definitions of $h_{i j}^{+}$and $h_{i j}^{-}$given in (3.4), it is possible to see that

$$
\begin{aligned}
& \left(\sum_{j=1}^{\mu_{B}} \sum_{i=1}^{\mu_{A}} \beta_{j} \alpha_{i} h_{l k}\left(v_{i j}\right)\right)^{+} \leq \sum_{j=1}^{\mu_{B}} \sum_{i=1}^{\mu_{A}} \beta_{j} \alpha_{i} h_{l k}^{+}\left(v_{i j}\right), \\
& \left(\sum_{j=1}^{\mu_{B}} \sum_{i=1}^{\mu_{A}} \beta_{j} \alpha_{i} h_{l k}\left(v_{i j}\right)\right)^{-} \leq \sum_{j=1}^{\mu_{B}} \sum_{i=1}^{\mu_{A}} \beta_{j} \alpha_{i} h_{l k}^{-}\left(v_{i j}\right),
\end{aligned}
$$


8 Control of constrained uncertain systems: a $\mathrm{pH}$ process

then

$$
\sum_{j=1}^{\mu_{B}} \sum_{i=1}^{\mu_{A}} \beta_{j} \alpha_{i} h_{l l}\left(\nu_{i j}\right) p_{1}^{l}+\sum_{\substack{k=1 \\ k \neq l}}^{m}\left(\sum_{j=1}^{\mu_{B}} \sum_{i=1}^{\mu_{A}} \beta_{j} \alpha_{i} h_{l k}\left(\nu_{i j}\right)\right)^{+} p_{1}^{k}+\sum_{\substack{k=1 \\ k \neq l}}^{m}\left(\sum_{j=1}^{\mu_{B}} \sum_{i=1}^{\mu_{A}} \beta_{j} \alpha_{i} h_{l k}\left(v_{i j}\right)\right)^{-} p_{2}^{k} \leq 0 .
$$

According to (2.5) and (2.6), the following result is obtained:

$$
H(q(t))=\sum_{j=1}^{\mu_{B}} \sum_{i=1}^{\mu_{A}} \beta_{j} \alpha_{i} H\left(v_{i j}\right)
$$

therefore

$$
h_{l l}(q(t)) p_{1}^{l}+\sum_{\substack{k=1 \\ k \neq l}}^{m} h_{l k}^{+}(q(t)) p_{1}^{k}+\sum_{\substack{k=1 \\ k \neq l}}^{m} h_{l k}^{-}(q(t)) p_{2}^{k} \leq 0
$$

hence

$$
H_{1}(q(t)) p_{1}+H_{2}(q(t)) p_{2} \leq 0 .
$$

Using a similar reasoning, it is possible to prove that (4.5) leads to

$$
H_{2}(q(t)) p_{1}+H_{1}(q(t)) p_{2} \leq 0,
$$

which gives the sufficient condition

$$
\tilde{H}(q(t)) P \leq 0
$$

Consequently, the set $\mathscr{D}_{P}$ is positively invariant with respect to motion of system (4.2) for all $q(t) \in \Gamma$.

Now, it is possible to make the desired extension. Hence, let us consider the nonautonomous uncertain system (2.1) with constrained control (2.2). The application of the feedback control law (2.9) leads to the closed-loop system (2.11).

Theorem 4.2. The subset $\mathscr{D}$ (2.15) is positively invariant with respect to motion of the uncertain system (2.11) with constrained control (2.2) if and only if there exist matrices $H\left(\nu_{i j}\right)$ for $i=1, \ldots, \mu_{A}, j=1, \ldots, \mu_{B}$, such that

$$
\begin{gathered}
F A_{c}\left(\nu_{i j}\right)=H\left(\nu_{i j}\right) F, \\
\tilde{H}\left(\nu_{i j}\right) U \leq 0 .
\end{gathered}
$$

Proof. Sufficiency. Assume that conditions (4.16) hold. By virtue of Theorem 3.2, the set $\mathscr{D}$ is positively invariant with respect to motion of system $(2.11)$ at the vertices $v_{i j}$ for $i=1, \ldots, \mu_{A}, j=1, \ldots, \mu_{B}$. Further, consider the following change of coordinates:

$$
z=F x
$$


the system (2.11) becomes system (4.2), and $\mathscr{D}$ is transformed as follows:

$$
\mathscr{D}_{z}=\left\{z \in \mathbb{R}^{m} /-u_{\min } \leq z \leq u_{\max }, u_{\min }, u_{\max } \in \mathbb{R}_{+}^{m}\right\} \text {, }
$$

hence $\mathscr{D}_{z}$ is positively invariant with respect to motion of system (4.2) at the vertices $v_{i j}$, for $i=1, \ldots \mu_{A}, i=1, \ldots, \mu_{B}$. Bearing in mind Lemma $4.1, \mathscr{D}_{z}$ is positively invariant with respect to system (4.2) for all $q \in \Gamma$. Consequently, $\mathscr{D}$ is positively invariant with respect to system (2.11) $\forall q \in \Gamma$.

Necessity. Let $\mathscr{D}$ be positively invariant with respect to system (2.11), so it is positively invariant at the vertices by using Lemma 4.1. Therefore, by virtue of Theorem 3.2, there exist matrices $H\left(\nu_{i j}\right)$ satisfying conditions (4.16) for every $i=1, \ldots, \mu_{A}, j=1, \ldots, \mu_{B}$.

Remark 4.3. For the sequel, and without loss of generality, assume that the system is square, that is, $n=m$. In fact if the system has $n$ states and $m$ inputs, $m \leq n$, then the system is augmented with constrained fictitious inputs $v[4]$. The resulting system is then given by

$$
\dot{x}(t)=A(q(t)) x(t)+B_{a}(q(t))\left[\begin{array}{l}
u(t) \\
v(t)
\end{array}\right],
$$

$B_{a}(q(t))$ is the matrix $B(q(t))$ augmented by $(n-m)$ null columns.

Corollary 4.4. The positive invariance of domain D with respect to motions of system (4.20) with state-feedback matrix F satisfying condition (2.9) implies the asymptotic stability of system (4.20) for every $x(0) \in \mathscr{D}$.

Proof. Assume that domain $\mathscr{D}$ is positively invariant with respect to motion of system (4.20), then by virtue of the Theorem 4.2 and using the Lemma 4.1, we have

$$
\begin{gathered}
F A_{c}(q(t))=H(q(t)) F, \\
\tilde{H}(q(t)) U \leq 0,
\end{gathered}
$$

then using Theorem 3.3, the nonsymmetric nonquadratic function

$$
V(x)=\operatorname{Max}_{l} \max \left\{\frac{(F x)_{l}^{+}}{u_{\max }^{l}}, \frac{(F x)_{l}^{-}}{u_{\min }^{l}}\right\}
$$

is a common Lyapunov function of all stationary configuration of the uncertain system (2.11) for every $q \in \Gamma$. Hence, the system (2.11) is asymptotically stable.

\section{Application to a $\mathrm{pH}$ process}

Consider the $\mathrm{pH}$ control plant shown in Figure 5.1 (already presented in $[29,30]$ ), which consists of a stirred tank where a solution of high concentration of the acid $\mathrm{ClH}$ is mixed with water to obtain a liquid of controlled $\mathrm{pH}$. The mixture's $\mathrm{pH}$ is measured using a $\mathrm{pH}-$ meter (Kent EIL9143), which presents appreciable inertia. Water is fed from a tank using a peristaltic pump, which produces a variable flow depending on the level of the liquid in 


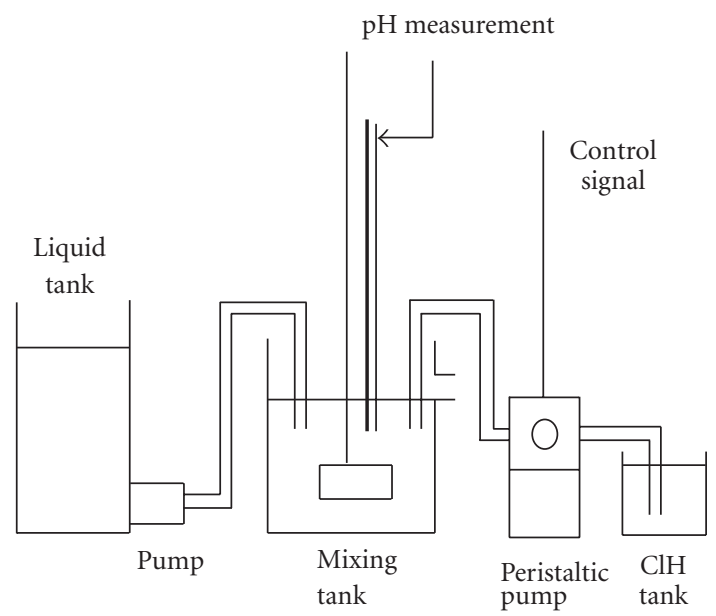

Figure 5.1. Laboratory plant.

the tank (Figure 5.1). Variations in the dynamics due to changing flows, concentrations, and operating points make this system uncertain.

It can be seen in Figure 5.1 that the control scheme in this plant is based on acid flow control. However the techniques presented now could be directly applied to other control structures such as base flow control [28], base flow control in the presence of buffer flow [6], or base and acid flow controls [13].

Although the modeling of $\mathrm{pH}$-control processes is well studied [15], in our case it is only necessary to have a simplified model, based on first principles. Assuming that the input liquid is pure water, that the acid solution has constant concentration on $\mathrm{ClH}$ and there is perfect solution, mixing, and no buffering, the following model can be obtained [25]:

$$
\begin{gathered}
M \frac{d N_{d}}{d t}=-q_{o} N_{d}-q_{a} N_{d}+q_{a} N_{a} \\
\tau \frac{d N_{d}^{*}}{d t}=N_{d}-N_{d}^{*}, \\
\mathrm{pH}=-\log _{10}\left(N_{d}^{*}\right),
\end{gathered}
$$

where $\tau$ is the measurement time constant, $M$ is the mass of the liquid in the tank, $q_{a}$ is the acid mass flow, $q_{o}$ is the liquid mass flow, $N_{d}$ is the acid concentration in the tank, $N_{d}^{*}$ is the measured concentration, and $N_{a}$ is the input acid concentration. The objective of the control system is to maintain the $\mathrm{pH}$ of the liquid in the mixing tank on desired values, using the acid mass flow $(u)$ as the control variable. The model parameters were estimated using measured data. 
The system can be represented by the following transfer function:

$$
G(s)=\frac{k}{(s-a)(s-b)},
$$

where the gain $k$ and the time constant $a$ are uncertain and the time constant $b$ is supposed constant. Uncertainty in the plant was experimentally estimated: different experiments under the most extreme conditions were done (maximum and minimum flows, extreme values of $\mathrm{pH}$, etc.) then the parameters were measured, using additional sensors where it was necessary (e.g., to evaluate the inlet flow range). The model was then linearized at these extreme points, and the extreme variations of gain and time constants selected as uncertainty in the model.

The system can be represented in state space by

$$
A=\left[\begin{array}{cc}
0 & 1 \\
-b a & b+a
\end{array}\right], \quad B=\left[\begin{array}{l}
0 \\
k
\end{array}\right]
$$

with constrained control $u$ in $\Omega$ given by (2.2), where $u_{\max }=60$, and $u_{\min }=35$.

It is experimentally known that the parameter $b$ can be assumed to be constant $(b=$ $-0.012725)$, as it suffers small variations which do not affect significantly the plant behavior. On the other hand, parameters $a$ and $k$ change greatly between working points, so they represent the parametric uncertainty. The parameter variations experimentally obtained were

$$
\begin{gathered}
k \in\left[-0.4649 \times 10^{-4},-0.7449 \times 10^{-4}\right], \\
a \in[-0.25,-2] .
\end{gathered}
$$

Let

$$
a_{0}=\frac{a_{\max }+a_{\min }}{2} \quad\left(\text { resp., } k_{0}=\frac{k_{\max }+k_{\min }}{2}\right),
$$

then any $a \in\left[a_{\min }, a_{\max }\right]$ (resp., $\left.k \in\left[k_{\min }, k_{\max }\right]\right)$ can be written as $a=a_{0}+q_{A}$ (resp., $\left.k=k_{0}+q_{B}\right)$, where $-\delta a \leq q_{A} \leq \delta a$ (resp., $-\delta k \leq q_{B} \leq \delta k$ ) with

$$
\delta a=\frac{a_{\max }-a_{\min }}{2} \quad\left(\text { resp. }, \delta k=\frac{k_{\max }-k_{\min }}{2}\right) .
$$

So, the form of the system becomes as (2.1), with

$$
A\left(q_{A}(t)\right)=\left[\begin{array}{cc}
0 & 1 \\
-b\left(a_{0}+q_{A}\right) & b+a_{0}+q_{A}
\end{array}\right], \quad B\left(q_{B}(t)\right)=\left[\begin{array}{c}
0 \\
k_{0}+q_{B}
\end{array}\right] .
$$

The vertices of domain of uncertainties are given by

$$
\begin{array}{cc}
v_{11}=\left(0.875,1.41 \times 10^{-5}\right), & v_{12}=\left(0.875,-1.41 \times 10^{-5}\right), \\
v_{21}=\left(-0.875,1.41 \times 10^{-5}\right), & v_{22}=\left(-0.875,-1.41 \times 10^{-5}\right) .
\end{array}
$$


The nominal system can be given by (2.10), where

$$
A_{0}=\left[\begin{array}{cc}
0 & 1 \\
-b a_{0} & b+a_{0}
\end{array}\right], \quad B_{0}=\left[\begin{array}{c}
0 \\
k_{0}
\end{array}\right] .
$$

Therefore, to assign two new eigenvalues to the closed-loop system, and without loss of generality, the system is augmented with fictitious constrained input $v$. This augmented system is given by

$$
\dot{x}(t)=A_{0} x(t)+B_{0 a}\left[\begin{array}{l}
u \\
v
\end{array}\right],
$$

$B_{0 a}$ is the matrix $B_{0}$ augmented by $(n-m)$ null columns, that is,

$$
B_{0 a}=\left[\begin{array}{cc}
0 & 0 \\
k_{0} & 0
\end{array}\right] .
$$

Constraints on the fictitious control $v$ are given by (2.2) with $v_{\max }=40$, and $v_{\min }=50$. In order to design a stabilizing controller for the nominal system, we use the so-called "inverse procedure" [1]. For this the matrix,

$$
H_{0}=\left[\begin{array}{cc}
-0.0130 & -0.0070 \\
0 & -1.1245
\end{array}\right]
$$

is selected satisfying conditions (3.2), then the algebraic equation $X A_{0}+X B_{0 a} X=H_{0} X$ is solved. Resolution of this equation leads to the following augmented regulator:

$$
F_{a}=10^{3}\left[\begin{array}{cc}
0.0050 & -0.0037 \\
-0.0170 & -1.3107
\end{array}\right] .
$$

Matrices $H\left(\nu_{i j}\right)$ for $i=1,2, j=1,2$ can be calculated as solutions of the equations $F_{a} A\left(\nu_{i}\right)+$ $F_{a} B_{a}\left(\nu_{j}\right) F_{a}=X\left(\nu_{i j}\right) F_{a}$. It is easy to check that condition (4.17), that is,

$$
\tilde{H}\left(v_{i j}\right) U_{a} \leq 0, \quad U_{a}=\left[\begin{array}{llll}
u_{\max }^{T} & v_{\max }^{T} & u_{\min }^{T} & v_{\min }^{T}
\end{array}\right]^{T},
$$

is satisfied for all $v_{i j}, i=1,2 ; j=1,2$. Hence, the computed regulator $F_{a}$ is robust. Notice here that for the effective control, the feedback signal can be extracted from $F_{a}$ as

$$
F=\left[\begin{array}{ll}
5 & -3.7
\end{array}\right]
$$

Figure 5.2 shows the evolution of the uncertain parameters $a$ and $k$ in time, the state vector evolution form initial condition $x_{o}=\left[\begin{array}{ll}0.02 & 0.03\end{array}\right]^{T}$, and the control evolution. It can be seen that robust regulation is achieved with respected control constraints.

\section{Conclusions}

This work has presented a solution to a control problem frequently found in process control, specially in $\mathrm{pH}$ control applications: the design of a linear controller for an uncertain 


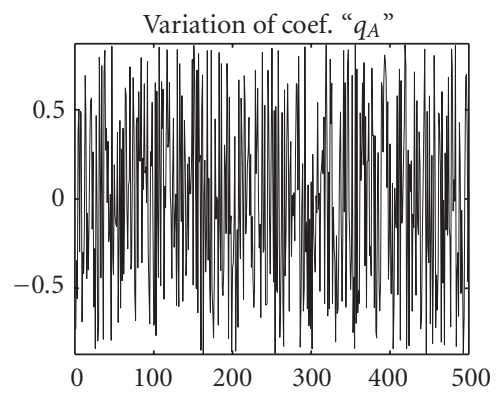

(a)

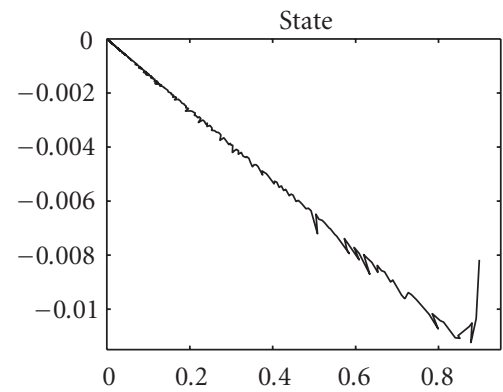

(c)

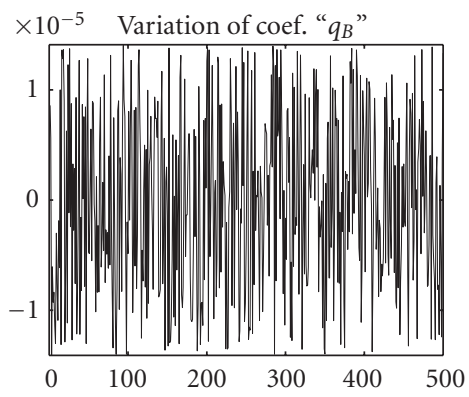

(b)

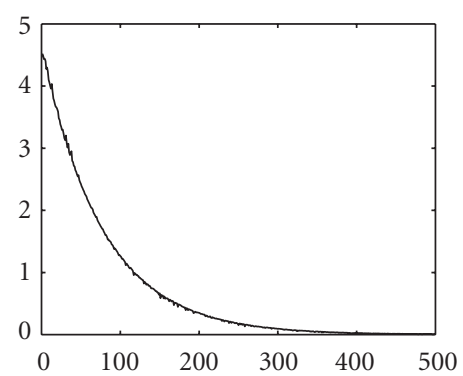

(d)

Figure 5.2. Simulation results.

plant with constrained inputs. This solution is based on using the concept of positive invariance for linear systems, extending this concept to the uncertain continuous-time case. Hence, necessary and sufficient conditions are derived enabling the designer to construct robust constrained controllers. It has been shown that those controllers can be designed by selecting the desired closed-loop performance, followed by computing the solution of an algebraic equation for the nominal system, and, finally checking the conditions of robustness at the vertices of the convex set of perturbations.

As an example, a laboratory $\mathrm{pH}$ process has been studied, showing that it is possible to model the uncertainty from a first-principle model. Based on this uncertainty and the physical constraints, the application of the method to calculate a robust controller has been shown. Application of this regulator to the $\mathrm{pH}$ process leads to asymptotic stability with admissible robust control. It must be pointed out that although the proposed technique was prompted by the $\mathrm{pH}$ control problem, the technique can also be applied to a wide area of practical control problems where constraints are present and the linear model is not perfectly known, such as many systems in process control, robotics, flight control, irrigation, and so forth.

Finally, it can be argued that the choice of matrix $H$ in this technique is done by trial and error: this can be restrictive and can generate a large computational load. However, it introduces a large degree of freedom that can be very useful for the control engineering 


\section{Control of constrained uncertain systems: a $\mathrm{pH}$ process}

in the sense that any spectrum can be assigned by this choice: by changing matrix $H$, directions of the pole assignment are changed, and different closed-loop performances can be achieved [5].

\section{References}

[1] A. Benzaouia, The resolution of equation $X A+X B X=H X$ and the pole assignment problem, IEEE Transactions on Automatic Control 39 (1994), no. 10, 2091-2095.

[2] A. Benzaouia and C. Burgat, Regulator problem for linear discrete-time systems with nonsymmetrical constrained control, International Journal of Control 48 (1988), no. 6, 2441-2451.

[3] A. Benzaouia and A. Hmamed, Regulator problem for linear continuous-time systems with nonsymmetrical constrained control, IEEE Transactions on Automatic Control 38 (1993), no. 10, $1556-1560$.

[4] A. Benzaouia and F. Mesquine, Regulator problem for uncertain linear discrete-time systems with constrained control, International Journal of Robust and Nonlinear Control 4 (1994), no. 3, 387395.

[5] A. Benzaouia, F. Mesquine, M. Naib, and A. Hmamed, Robust pole assignment in complex plane regions for linear uncertain constrained control systems, International Journal of Systems Science 32 (2001), no. 1, 83-89.

[6] K. K. Biasizzo, I. Skrjanc, and D. Matko, Fuzzy predictive control of highly nonlinear pH process, Computers \& Chemical Engineering 21 (1997), no. 1-2, S613-S618.

[7] G. Bitsoris and E. Gravalou, Robust linear controller under state and control constraints, Proceedings of Conference on Decision and Control, Arizona, 1992.

[8] F. Blanchini, Feedback control for linear time-invariant systems with state and control bounds in the presence of disturbances, IEEE Transactions on Automatic Control 35 (1990), no. 11, 1231-1234.

[9] __ Set invariance in control, Automatica 35 (1999), no. 11, 1747-1767.

[10] E. F. Camacho and C. Bordons, Model Predictive Control, Springer, London, 1999.

[11] M. A. Dahleh and I. J. Diaz-Bobillo, Control of Uncertain Systems: A Linear Programming Approach, Prentice-Hall, New Jersey, 1995.

[12] M. J. Fuente, C. Robles, O. Lobato, and F. Tadeo, Fuzzy control of a neutralization process, Proceedings of IEEE Conference on Control Applications, Glasgow, 2002, pp. 1032-1037.

[13] O. Galan, J. A. Romagnoli, and A. Palazoglu, Robust $H_{\infty}$ control of nonlinear plants based on multi-linear models: an application to a bench-scale pH neutralization reactor, Chemical Engineering Science 55 (2000), no. 20, 4435-4450.

[14] J. B. Gomm, S. K. Doherty, and D. Williams, Control of $\mathrm{pH}$ in-line using a neural predictive strategy, Proceedings of IEE UKACC International Conference on Control, Rhode Island, 1996.

[15] T. F. Gustafsson and K. V. Waller, Nonlinear and adaptive control of $p H$, Industrial and Engineering Chemistry Research 24 (1992), 809-817.

[16] D. Henrion, S. Tarbouriech, and V. Kučera, Control of linear systems subject to input constraints: a polynomial approach, Automatica 37 (2001), no. 4, 597-604.

[17] Z. Lin and A. Saberi, Semi-global exponential stabilization of linear systems subject to "input saturation" via linear feedbacks, Systems \& Control Letters 21 (1993), no. 3, 225-239.

[18] J. M. Maciejowski, Predictive Control with Constraints, Prentice-Hall, New Jersey, 2001.

[19] F. Mesquine, A. Benlamkadem, and F. Tadeo, Robust constrained Regulator problem for linear uncertain continuous time systems: application to a $\mathrm{pH}$ process, Proceedings of the IEEE International Conference on Control Applications, Glasgow, 2002, pp. 391-396.

[20] F. Mesquine, A. Benlemkadem, and A. Benzaouia, Robust constrained regulator problem for linear uncertain systems, Journal of Dynamical and Control Systems 10 (2004), no. 4, 527-544.

[21] F. Mesquine, F. Tadeo, and A. Benzaouia, Regulator problem for linear systems with constraints on control and its increment or rate, Automatica 40 (2004), no. 8, 1387-1395. 
[22] N. R. L. Narayanan, P. R. Krishnaswamy, and G. P. Rangaiah, Use of alternate process variable for enhancing pH control performance, Chemical Engineering Science 53 (1998), no. 17, 3041-3049.

[23] S. J. Norquay, A. Palazoglu, and A. Romagnoli, Model predictive control based on Wiener models, Chemical Engineering Science 53 (1998), no. 1, 75-84.

[24] R. H. Nystrom, K. V. Sandstom, T. K. Gustafsson, and H. T. Toivonen, Multimodel robust control applied to a pH neutralization process, Computers \& Chemical Engineering 22 (1998), S467S474.

[25] O. Pérez, F. Tadeo, and P. Vega, Robust control of pH control plant, Proceedings of IEEE Conference on Control Applications, New York, 1995.

[26] C. H. Sing and B. Postlethwaite, pH control: handling nonlinearity and deadtime with fuzzy relational model-based control, IEE Proceedings-Control Theory and Applications 144 (1997), no. 3, 263-268.

[27] A. A. Stoorvogel, A. Saberi, and P. Sannuti, Performance with regulation constraints, Automatica 36 (2000), no. 10, 1443-1456.

[28] S. W. Sung and I. B. Lee, $p H$ control using a simple set point change, Industrial and Engineering Chemistry Research 34 (1995), no. 5, 1730-1734.

[29] F. Tadeo, A. Holohan, and P. Vega, $l_{1}$-optimal regulation of a $\mathrm{pH}$ control plant, Computers \& Chemical Engineering 22 (1998), S459-S466.

[30] F. Tadeo, O. P. Lopez, and T. Alvarez, Control of neutralization processes by robust loop shaping, IEEE Transactions on Control Systems Technology 8 (2000), no. 2, 236-246.

[31] S.-S. Yoon, T.-W. Yoon, D. R. Yang, and T.-S. Kang, Indirect adaptive nonlinear control of a pH process, Computers \& Chemical Engineering 26 (2002), no. 9, 1223-1230.

[32] K. Zhou, J. C. Doyle, and K. Glover, Robust and Optimal Control, Prentice-Hall, New Jersey, 1996.

Fouad Mesquine: Constrained and Robust Regulation Research Unit, Physics Department, Faculty of Sciences, Cadi Ayyad University, B.P. 2390, Marrakesh, Morocco

E-mail address: mesquine@ucam.ac.ma

Fernando Tadeo: Departamento de Ingeniería de Sistemas y Automática, Universidad de Valladolid, 47005 Valladolid, Spain

E-mail address: fernando@autom.uva.es

Abdellatif Benlamkadem: Constrained and Robust Regulation Research Unit, Physics Department, Faculty of Sciences, Cadi Ayyad University, B.P. 2390, Marrakesh, Morocco 


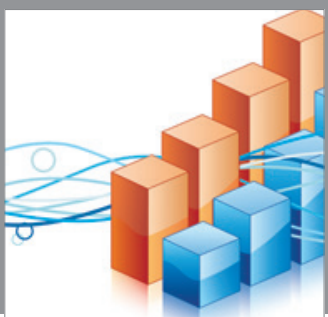

Advances in

Operations Research

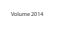

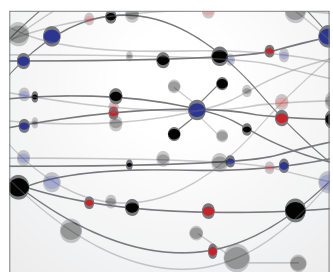

\section{The Scientific} World Journal
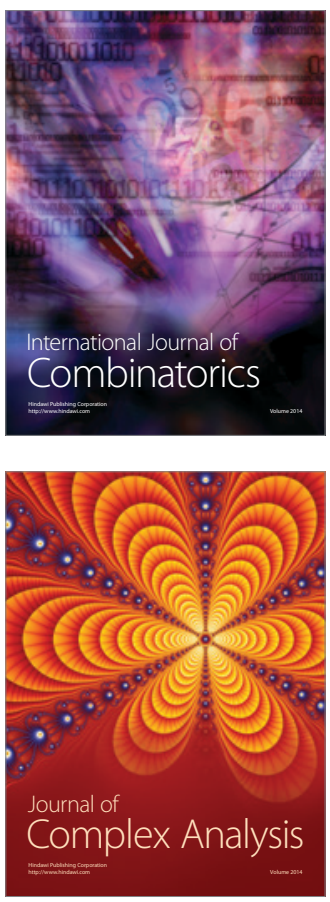

International Journal of

Mathematics and

Mathematical

Sciences
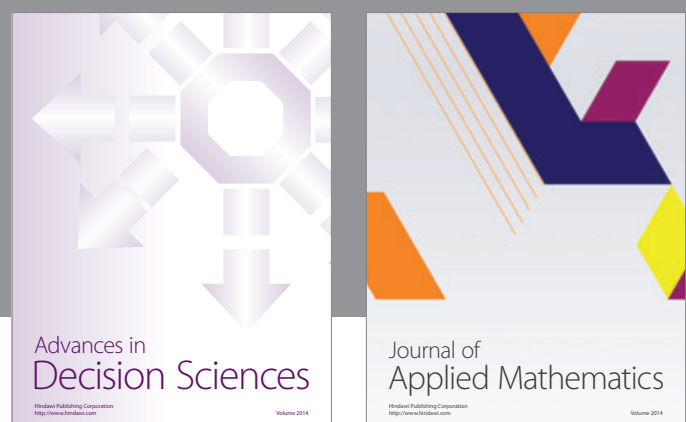

Journal of

Applied Mathematics
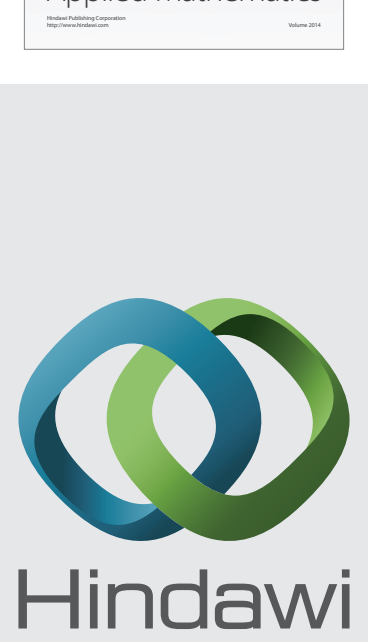

Submit your manuscripts at http://www.hindawi.com
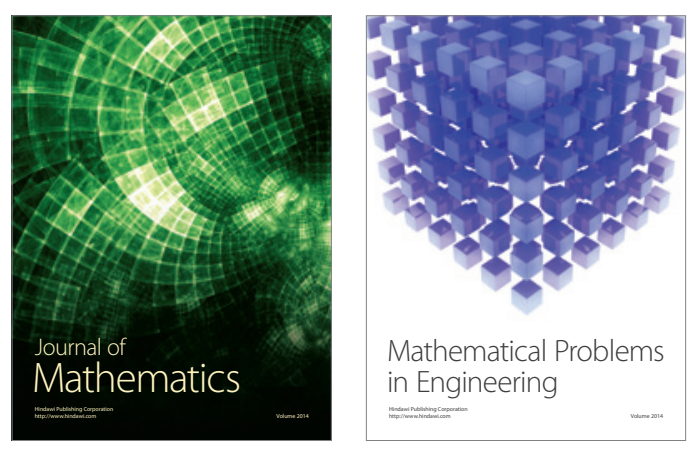

Mathematical Problems in Engineering
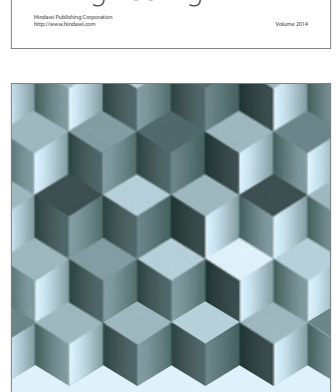

Journal of

Function Spaces
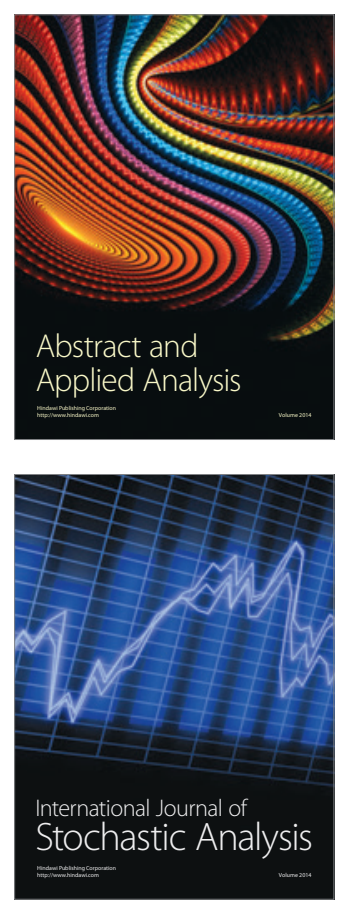

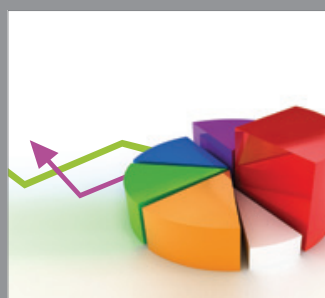

ournal of

Probability and Statistics

Promensencen
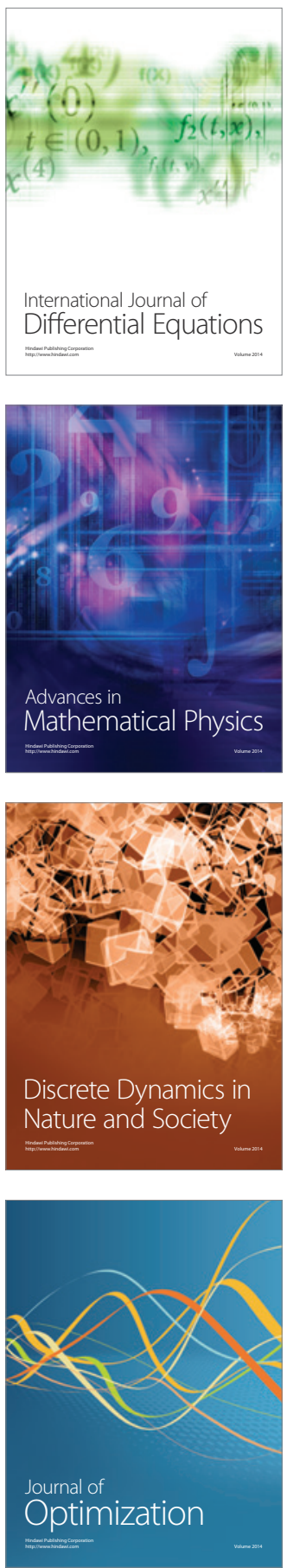